\title{
Peanuts in barred analytic potentials
}

\author{
Patsis P.A., Skokos Ch. \\ Research Center for Astronomy, Academy of Athens, Anagnostopoulou \\ 14, GR-106 73, Greece \\ Athanassoula E. \\ Obseratoire de Marseille, 2 Place Le Verrier, F-13248, Marseille Cedex \\ 4, France
}

\begin{abstract}
We investigate the orbital behavior in a class of analytic potentials in order to isolate the families of orbits which lead to the appropriate appearance of boxy/peanut features. This is the first step towards understanding the orbits of particles in $N$-body simulations of barred galaxies. Our final goal is to find out which dynamical mechanisms determine the peanut or boxy shapes.
\end{abstract}

We used families, and sets of families, of periodic orbits in order to evaluate their importance as building blocks for boxy and peanut (hereafter $b / p$ ) shapes. We find cases where the $b / p$ profile is confined to the central parts of the model and cases where the whole bar participates in this morphology. The peanut can be built not only by $3 \mathrm{D}$ families associated with the vertical $2: 1$ resonance or other $3 \mathrm{D}$ bifurcations of $\mathrm{x} 1$ at higher order resonances, but even by families related to the z-axis orbits existing over large energy intervals. The observed ' $X$ ' feature inside the boxy bulges of some edge-on galaxies can be attributed to the peaks of successive 3D orbits of specific families. There are also other families which exist over large energy intervals and whose projections pass through the center of the galaxy. They support a b/p but not necessarily the ' $X$ ' feature. The detailed morphology of the two cases differs. Families being complex unstable (Contopoulos \& Magnenat 1985) in large energy intervals have difficulties to be organized in a b/p structure. The present study favors the idea that the observed edge-on profiles are the imprints of families of periodic orbits in appropriately chosen Hamiltonian systems, describing the potential of the bar.

Our main conclusions are:

1. The vertical profiles of our models are of 'stair-type'. The closer to corotation a $3 \mathrm{D}$ family supporting the bar is born the lower its main height is.

2. The projection of the families has a limited extent on the equatorial plane. This means that the $3 \mathrm{D}$ families bifurcated from $\mathrm{x} 1$ at a vertical resonance build isolated dynamical blocks and cannot account for the whole observed vertical profile in a galaxy. Especially those that are bifurcated at low order 


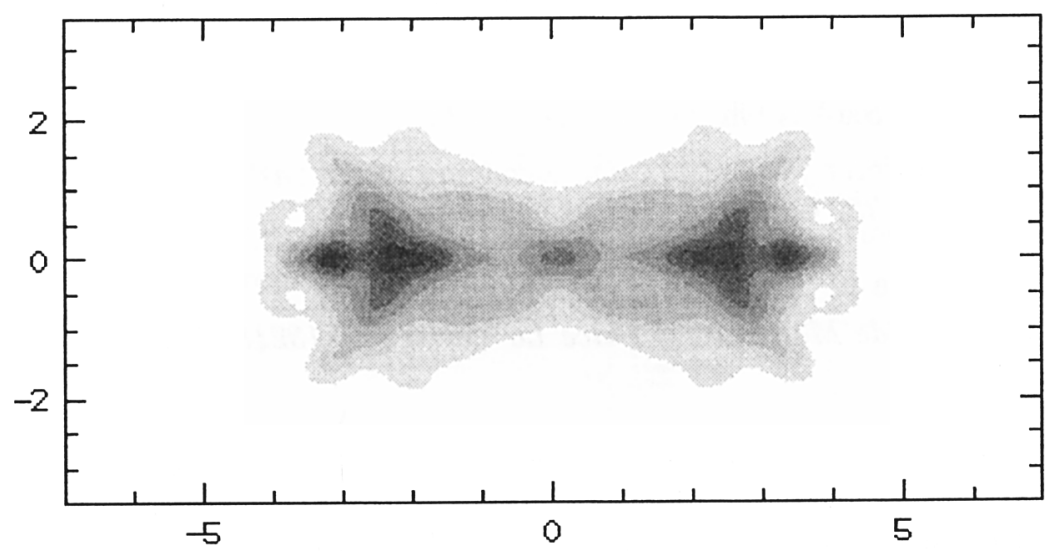

Figure 1. An image of a side-on orbital profile of a model consisting of a 3D Ferrers bar and a Miyamoto disk. The profile is built by the superposition of the orbits of three families, two of which are vertical bifurcations of $\mathrm{x} 1$ and the third is related with the z-axis orbits. The composite effect is a strong peanut morphology. The image is blurred in order to underline the coarse morphological features.

resonances, e.g. at the vertical $2: 1$, can be responsible for $a \mathrm{~b} / \mathrm{p}$ feature but not for a thick bar with a $b / p$ edge-on structure.

3. The families supporting the peanut can be either $3 \mathrm{D}$ bifurcations of the $\mathrm{x} 1$ family or, in some specific cases, families related with the $\mathrm{z}$-axis orbits, i.e. the family with orbits on the rotational axis.

4. Orbital profiles favor the appearance of characteristic local enhancements of the surface density along the major axis of the bar as observed in many edge-on disk galaxies.

This dynamical behavior will be compared to the orbital dynamics in $N$ body simulations.

\section{References}

Contopoulos G., Magnenat P., 1985, Celest. Mech. 37, 387

Acknowledgments. This work has been done in the frame of the project

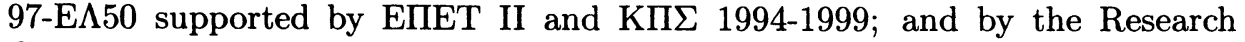
Committee of the Academy of Athens. 